\title{
BMJ
}

\section{Impact of a new national screening policy for Down's syndrome in Denmark: population based cohort study}

\author{
Charlotte K Ekelund, research assistant, ${ }^{1}$ Finn Stener Jørgensen, consultant, ${ }^{2}$ Olav Bjørn Petersen, \\ consultant, ${ }^{3}$ Karin Sundberg, consultant, ${ }^{1}$ Ann Tabor, professor, ${ }^{1,4}$ Danish Fetal Medicine Research Group
}

${ }^{1}$ Department of Fetal Medicine, Rigshospitalet, Copenhagen University Hospital, 2100 Copenhagen, Denmark

${ }^{2}$ Fetal Medicine Unit, Department of Obstetrics and Gynaecology, Hvidovre University Hospital, Denmark

${ }^{3}$ Department of Obstetrics and Gynaecology, Skejby University Hospital, Aarhus, Denmark

${ }^{4}$ Faculty of Health Sciences, University of Copenhagen

Correspondence to: A Tabor ann.tabor@rh.regionh.dk

Cite this as: BMJ 2008;337:a2547 doi:10.1136/bmj..22547

\section{ABSTRACT}

Objectives To evaluate the impact of a screening strategy in the first trimester, introduced in Denmark during 20046 , on the number of infants born with Down's syndrome and the number of chorionic villus samplings and amniocenteses, and to determine detection and false positive rates in the screened population in 2005 and 2006.

Design Population based cohort study.

Setting 19 Danish departments of gynaecology and obstetrics and a central cytogenetic registry 2000-7. Participants 65000 pregnancies per year.

Main outcome measures The primary outcomes measured were number of fetuses and newborn infants with Down's syndrome diagnosed prenatally and postnatally and number of chorionic villus samplings and amniocenteses carried out. Secondary outcomes measured were number of women screened in 2005 and 2006, screen positive rate, and information on screening in 2005 and 2006 for infants with a postnatal diagnosis of Down's syndrome. Results The number of infants born with Down's syndrome decreased from 55-65 per year during 2000-4 to 31 in 2005 and 32 in 2006. The total number of chorionic villus samplings and amniocenteses carried out decreased from 7524 in 2000 to 3510 in 2006. The detection rate in the screened population in 2005 was $86 \%$ (95\% confidence interval $79 \%$ to $92 \%$ ) and in 2006 was $93 \%$ ( $87 \%$ to $97 \%$ ). The corresponding false positive rates were $3.9 \%$ (3.7\% to $4.1 \%$ ) and $3.3 \%$ (3.1\% to $3.4 \%$ ).

Conclusion The introduction of a combined risk assessment during the first trimester at a national level in Denmark halved the number of infants born with Down's syndrome. The strategy also resulted in a sharp decline in the number of chorionic villus samplings and amniocenteses carried out, even before full implementation of the policy.

\section{INTRODUCTION}

In September 2004 the Danish National Board of Health issued new guidelines for prenatal screening and diagnosis. ${ }^{1}$ These recommended that pregnant women should be offered information about screening methods in pregnancy and, if desired, a combined risk assessment for Down's syndrome in the first trimester based on a combination of maternal age, nuchal translucency scanning, and a biochemical test for serum free $\beta$ human chorionic gonadotrophin and pregnancy associated plasma protein $\mathrm{A}$, called the double test. On the basis of this assessment women were to be informed about their risk (given as odds, such as 1:1250) of carrying a fetus with Down's syndrome. Women with a risk above a defined cut-off (for example, 1:300) were to be offered an invasive diagnostic procedure (chorionic villus sampling or amniocentesis). According to the previous guidelines from the Danish National Board of Health, pregnant women were to be offered chorionic villus sampling or amniocentesis if they were aged 35 or more, were at increased risk of carrying a fetus with Down's syndrome on the basis of serum screening using a triple test in the second trimester, or were at risk of an inherited disease. In 2000 the uptake of invasive diagnostic testing in women aged 35 or more was less than $50 \%$, whereas around $20 \%$ of all pregnant women had nuchal translucency ultrasonography. ${ }^{2}$ The triple test was not offered to all women but was done in about $10 \%$ of the population. Scans for malformations in the second trimester were offered to $28 \%$ of women. ${ }^{2}$

All 15 Danish counties decided to follow the guidelines from 2004 and introduce combined risk assessment in the first trimester. The cost of introducing the programme (ultrasound and laboratory equipment, training, wages for new staff) was covered by the counties and local hospitals. In 2004-6 the risk cut-off for referral to invasive diagnostic procedures varied between counties, from 1:250 to 1:400. The new policy was expected to detect $90 \%$ of fetuses with Down's syndrome at a $5 \%$ false positive rate on the basis of calculations made on the Danish population in 2001.

We evaluated the impact at a national level of the introduction of this new screening strategy on the number of infants born with Down's syndrome and on the number of chorionic villus samplings and amniocenteses. We also assessed whether the detection and false positive rates in the screened population for 2005 and 2006 were as expected.

\section{METHODS}

Denmark has a population of 5.4 million primarily white people and about 65000 liveborn infants per 


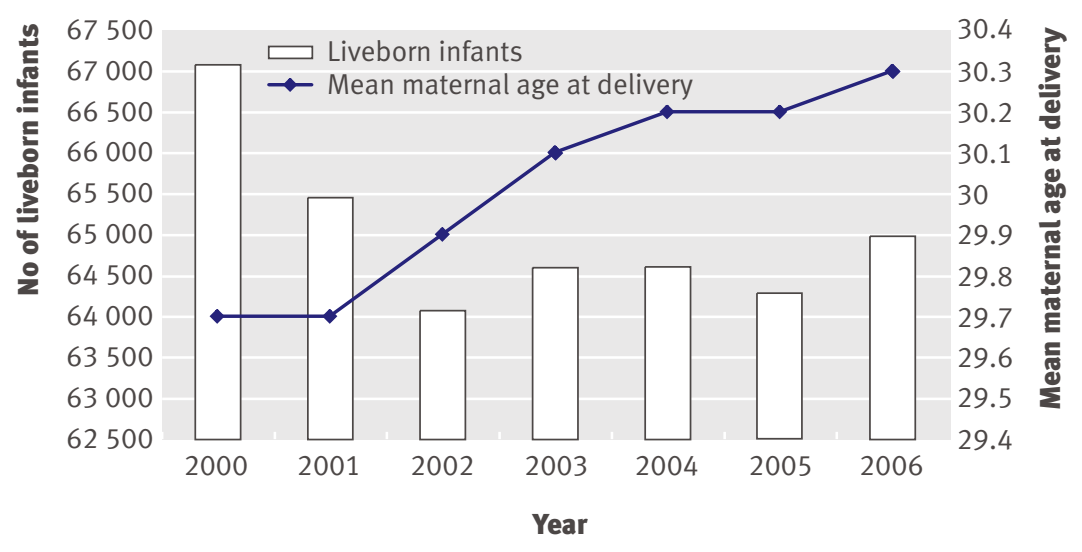

Fig 1| Number of liveborn infants and mean maternal age at delivery in Denmark, 2000-6

year (www.statistikbanken.dk). At birth everyone is assigned a unique personal registration number, which is used for identification in the Danish social and healthcare system. This centralised, computer based, registration system enables follow-up of individuals through public registries.

From Statistics Denmark (www.statistikbanken.dk) we retrieved data on the number of liveborn infants born per year, the distribution of maternal age at delivery, and the mean maternal age at delivery for the period 2000-6. Using the maternal age specific risk of delivering an infant with Down's syndrome we calculated the expected number of liveborn infants with Down's syndrome. ${ }^{3}$

In Denmark results from prenatal and postnatal chromosome analyses are forwarded to the Danish central cytogenetic registry. From there we obtained information on the number of chorionic villus samplings and amniocenteses carried out during 2000-6, the indications for either procedure, and karyotypes.

In Denmark all newborn infants are examined by a midwife. When an abnormality or malformation such as Down's syndrome is suspected, follow-up with a paediatrician is initiated. The results of postnatal chromosome analysis including the personal registration numbers of the mother and infant are sent to the Danish central cytogenetic registry. The registry provided information on the number of infants with Down's syndrome born during 2000-4 as well as the personal registration number of all infants with Down's syndrome born during 2005-7 and their mothers.

For various political and practical reasons one county (Funen) had not yet reported the results of their chromosome analyses to the registry. We therefore obtained information separately on the number of chorionic villus samplings and amniocenteses and prenatal and postnatal cases of Down's syndrome for 2000-6 from Funen's chromosome laboratory.

Nuchal translucency ultrasonography is carried out by nurses, midwives, and doctors certified by and in accordance with the guidelines of the Fetal Medicine Foundation in London (www.fetalmedicine.com/). All obstetrics and gynaecology departments in Denmark use the same fetal medicine software program (Astraia,
Germany) for calculating risk based on formulas derived by the Fetal Medicine Foundation. In some hospitals blood samples collected for the double test (serum free $\beta$ human chorionic gonadotrophin and pregnancy associated plasma protein A) are analysed at local laboratories, whereas other hospitals send samples to a central laboratory. Most of the laboratories use Brahms Kryptor (Brahms, Immunodiagnostic Systems, UK) for biochemical analyses and a few use an alternative immunoassay (Delfia Xpress; PerkinElmer, Waltham, MA).

Evaluation of screening performance in 2005 and 2006 From the 19 obstetrics and gynaecology departments we collected information on the number of women who had had a risk assessment for Down's syndrome in the first trimester in 2005 and 2006, either as the optimal combined test (maternal age, nuchal translucency scan, and biochemistry) or by a combination of maternal age and nuchal translucency scan or biochemistry. To enable us to evaluate the screen positive rate, the departments reported the number of women given a risk assessment of 1:300 or more at the time of screening. We chose this uniform cut-off to simplify the presentation of data, despite some departments using a slightly different cut-off for referral to invasive diagnostic testing.

In the calculation of screening performance we included fetuses and newborn infants with Down's syndrome when a first trimester screening test had been done in 2005 or 2006. Information about gestational age at delivery for all infants with Down's syndrome born during 2005-7 was obtained from the Danish National Board of Health.

We cross checked the personal registration numbers of women who had given birth to an infant with Down's syndrome during 2005-7 with all Astraia database servers in Denmark to obtain information on whether screening had been carried out in the first trimester. Information about screening was also requested in those cases where Down's syndrome was diagnosed prenatally by an invasive procedure carried out for indications other than an increased risk of Down's syndrome.

\section{RESULTS}

A combined risk assessment in the first trimester was introduced successively in Denmark. In January 2005

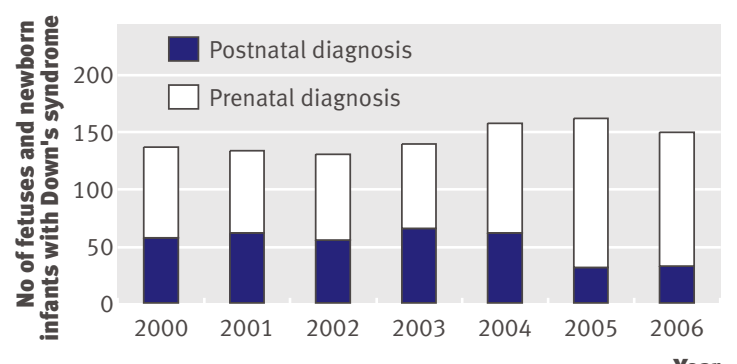

Fig 2 | Number of fetuses and newborn infants with Down's syndrome diagnosed prenatally or postnatally in Denmark, 2000-6 
nine of the 15 counties (60\%) offered women screening, increasing to 13 counties (87\%) by January 2006. By June 2006 the whole of the country was covered.

The yearly number of deliveries in Denmark decreased slightly during 2000-6 (fig 1), whereas the mean maternal age at delivery increased from 29.7 years in 2000 to 30.3 years in 2006 . Based on the actual distribution of maternal age and if no prenatal screening or invasive diagnosis had been carried out, the estimated expected number of infants with Down's syndrome increased from 121 in 2000 to 132 in 2005 and 135 in 2006.

\section{Number of newborn infants with Down's syndrome}

The number of newborn infants with Down's syndrome decreased from 55 -65 per year in 2000-4 to 31 in 2005 and 32 in 2006 . The total number of fetuses and newborn infants with Down's syndrome diagnosed

Data on screening variables from 19 pregnancies in 2005 and 11 in 2006 resulting in newborn infants with Down's syndrome in Denmark

\begin{tabular}{|c|c|c|c|}
\hline $\begin{array}{l}\text { Year, maternal age* } \\
\text { (years) }\end{array}$ & $\begin{array}{l}\text { Nuchal translucency } \\
\qquad(\mathrm{mm})\end{array}$ & $\begin{array}{l}\text { Biochemistry } \\
\text { performed }\end{array}$ & $\begin{array}{c}\text { Risk } \\
\text { assessment }\end{array}$ \\
\hline \multicolumn{4}{|l|}{ 2005: } \\
\hline 25 & 1.3 & Yes & $1: 23641$ \\
\hline 26 & 2.4 & Yes & $1: 793$ \\
\hline 28 & 1.5 & Yes & $1: 2838$ \\
\hline 28 & 1.5 & Yes & $1: 2598$ \\
\hline 29 & 1.7 & No & $1: 4954$ \\
\hline 29 & 1.8 & Yes & $1: 2980$ \\
\hline 30 & 1.9 & Yes & $1: 1831$ \\
\hline 30 & 1.8 & Yes & 1:195† \\
\hline 32 & 1.8 & Yes & $1: 627$ \\
\hline 32 & 1.8 & Yes & 1:3193 \\
\hline 34 & 2.3 & Yes & $1: 682$ \\
\hline 35 & Reported as "normal" & No & $1: 1229$ \\
\hline 35 & 2.0 & Yes & $1: 775$ \\
\hline 35 & 2.3 & Yes & $1: 64 \dagger$ \\
\hline 36 & 1.9 & Yes & $1: 3847$ \\
\hline 40 & 2.2 & Yes & $1: 672$ \\
\hline 40 & - & Yes & $1: 3 \ddagger$ \\
\hline 41 & 2.0 & Yes & $1: 466$ \\
\hline 46 & 2.0 & Yes & $1: 729$ \\
\hline \multicolumn{4}{|l|}{ 2006: } \\
\hline 24 & 1.6 & Yes & $1: 1707$ \\
\hline 25 & 3.0 & Yes & $1: 322$ \\
\hline 27 & 2.9 & Yes & $1: 492$ \\
\hline 30 & 1.8 & Yes & $1: 1764$ \\
\hline 31 & 1.7 & Yes & $1: 79 \dagger$ \\
\hline 33 & 1.8 & Yes & $1: 7693$ \\
\hline 34 & 1.5 & Yes & $1: 3246$ \\
\hline 35 & 3.0 & Yes & $1: 66 \dagger$ \\
\hline 37 & 6.3 & Yes & $1: 5 \dagger$ \\
\hline 37 & 2.3 & No (twins) & $1: 206 \dagger$ \\
\hline 40 & 2.3 & Yes & $1: 79 \dagger$ \\
\hline
\end{tabular}

*Maternal age at time of nuchal translucency scan. If no scan was done then maternal age at week $12+4$ is reported.

†Offered diagnostic testing.

$\ddagger$ Gestational age at screening $14+2$, offered diagnostic testing.

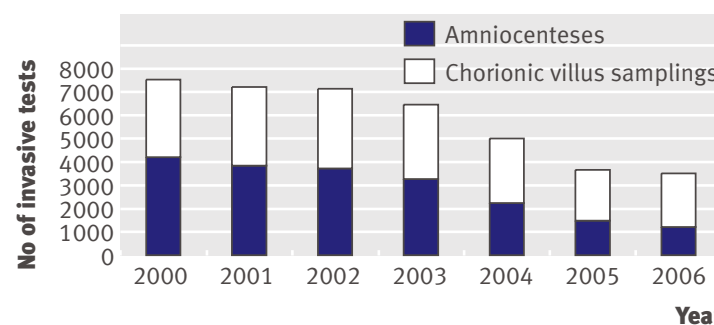

Fig 3 Number of amniocenteses and chorionic villus samplings carried out in Denmark, 2000-6

prenatally or postnatally in 2000-3 was stable at around $135-140$ per year, with an increase to 157 in 2004,161 in 2005, and 149 in 2006 (fig 2). The proportion of cases diagnosed prenatally increased from 53-61\% during $2000-4$, to $81 \%$ in 2005 and $79 \%$ in 2006 .

\section{Prenatal diagnostic procedure rate}

The number of prenatal diagnostic procedures (chorionic villus samplings or amniocenteses) decreased from 7524 in 2000 to 3510 in 2006 (fig 3). The number of chorionic villus samplings decreased from 3322 in 2000 to 2302 in 2006, while the number of amniocenteses carried out decreased from 4202 to 1208 in the same years. This corresponds to an increase in the proportion of chorionic villus samplings from $44 \%$ to $66 \%$.

\section{Screening performance in 2005 and 2006}

About 65000 women were pregnant in Denmark during 2005-6. In 200540815 women (62.8\%) had a risk assessment carried out in the first trimester, increasing to $54830(84.4 \%)$ in 2006. The remaining women had no risk assessment done because they were offered an invasive diagnostic test for reasons other than a screen positive test result, declined screening, or failed to receive an offer for reasons such as residency in a county not yet offering screening. Figures 4 and 5 show the distribution of women eligible for screening and the groups in which infants with Down's syndrome were diagnosed prenatally and postnatally.

In 2005 a total of 1706 women $(4.2 \%)$ had a risk of 1:300 or more (screen positive rate) and among these, 1388 women $(81.4 \%)$ decided to have a diagnostic test (fig 4). Seventy two per cent of the diagnostic procedures done because of a screen positive risk assessment were chorionic villus samplings, the remainder were amniocenteses. In 2006 a total of 1899 women $(3.5 \%)$ had a risk of $1: 300$ or more and $1704(89.7 \%)$ underwent diagnostic testing as a consequence of the screening result. Seventy six per cent of the diagnostic procedures carried out because of a screen positive risk assessment were chorionic villus samplings.

In the population screened in 2005 the detection rate of Down's syndrome was 86\% (95\% confidence interval $79 \%$ to $92 \%)-(101+3) /(101+3+16+1)$-as 104 of 121 women carrying a fetus with Down's syndrome were screened true positive (fig 4). Thus 17 
women received a false negative screening result. One of these women had an amniocentesis on suspicion of a malformation after the $18-20$ week scan, and the pregnancy was terminated (fig 4). An adjusted detection rate taking into account fetal loss from screening to time of birth (estimated as 25\% $\%^{4}$ ) was $82 \%(95 \%$ confidence interval $73 \%$ to $90 \%$ ). The false positive rate was $3.9 \%$ (3.7\% to $4.1 \%)$.

In 2006 the detection rate was $93 \%$ (87\% to $97 \%)$ $(92+5) /(92+5+6+1)$ — as only seven women received a false negative screening result. One of these women had an amniocentesis on suspicion of a malformation, and the pregnancy was terminated (fig 5). The adjusted detection rate taking fetal loss into account was estimated at $92 \%(83 \%$ to $97 \%)$. The false positive rate was $3.3 \%(3.1 \%$ to $3.4 \%)$.

The odds of being affected (carrying a fetus with Down's syndrome) after receiving a screen positive risk assessment during the first trimester were $1: 16$ in 2005 and 1:20 in 2006. The odds of being affected after receiving a screen negative result were 1:2301 in 2005 and 1:7562 in 2006.

The odds of being affected after undergoing chorionic villus sampling or amniocentesis owing to advanced maternal age were similar in 2005 and 2006 (1:65 and 1:75); 15 fetuses with Down's syndrome were diagnosed among 980 women in 2005 and eight fetuses among 600 women in 2006. Indications other than advanced maternal age or high risk after screening for undergoing chorionic villus sampling or amniocentesis were mainly family history of chromosomal abnormality, mental retardation or monogenic inherited disease, or a high risk on the basis of serum screened in the second trimester.

Thirty infants with Down's syndrome were born to mothers who had had a risk assessment done in the first trimester during 2005 and 2006. The table gives the details of the risk assessments.

\section{DISCUSSION}

Even before full implementation of the policy for combined risk assessment during the first trimester in Denmark, the number of infants born with Down's syndrome decreased by about $50 \%$ and the number of cases diagnosed prenatally increased by around 30\%.

The number of fetuses and newborn infants with Down's syndrome diagnosed prenatally or postnatally increased in the period 2000-5, with a slight decline in 2006 (fig 2). This was partly due to increasing maternal age, but was as expected because more fetuses with Down's syndrome are lost spontaneously than those that are chromosally normal. This increased rate has been estimated at around 25\% from week 14 to term. ${ }^{4}$ Based on the known distribution of maternal age at delivery in 2005 and 2006, 132 and 135 infants with Down's syndrome would have been expected in our population of 65000 liveborn infants if the mothers had no prenatal intervention. Down's syndrome was diagnosed in 31 infants postnatally and 130 prenatally in 2005 and in 32 infants postnatally and 117 prenatally in 2006. Given a rate for fetal loss of $25 \%$, this corresponds to 129 infants with Down's syndrome diagnosed postnatally in 2005 and 120 diagnosed postnatally in 2006. In 2005 the expected numbers

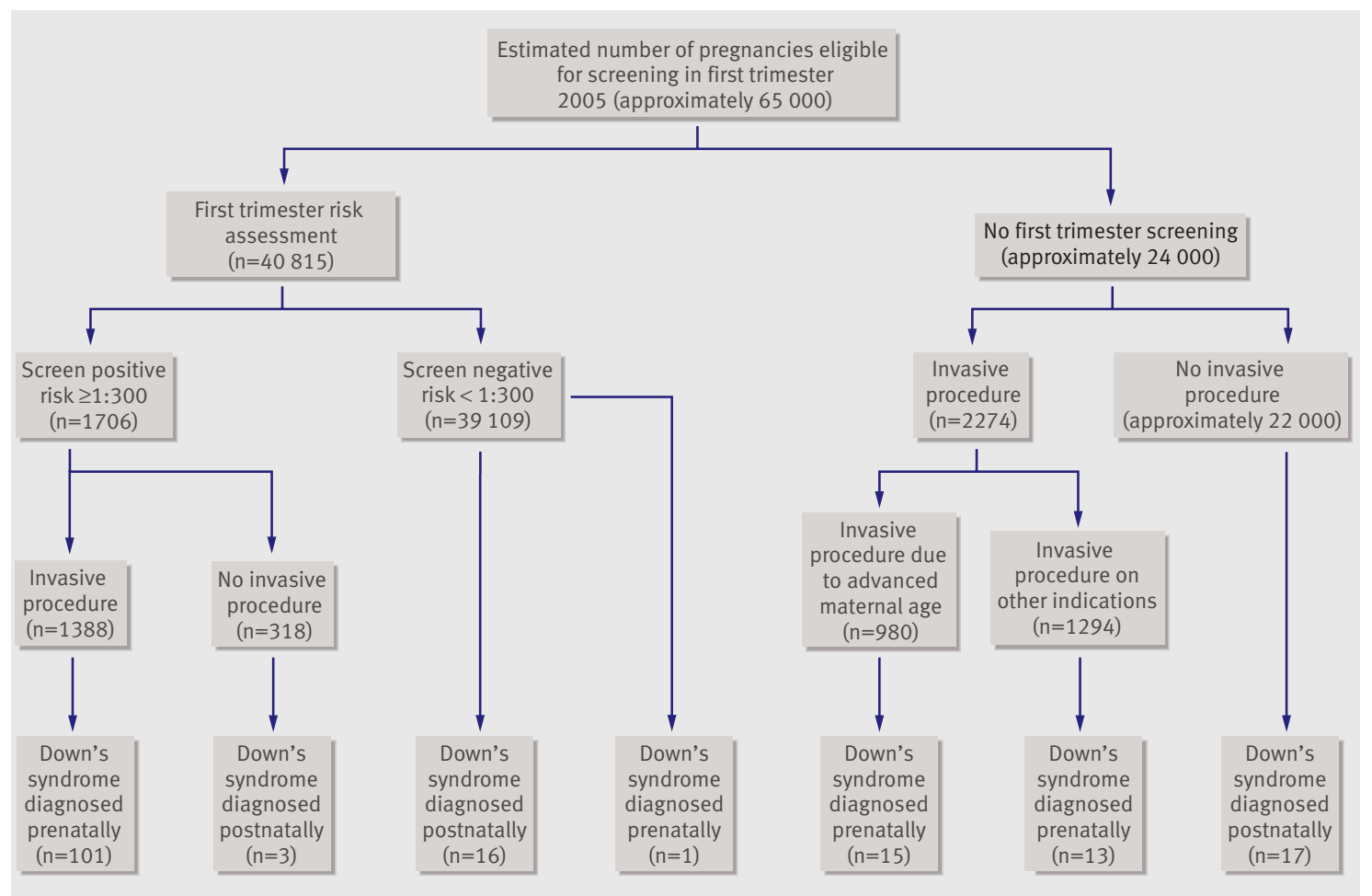

Fig 4 Number of fetuses and newborn infants with Down's syndrome diagnosed prenatally or postnatally according to screening results in Denmark, 2005. Invasive procedures are chorionic villus samplings or amniocenteses 


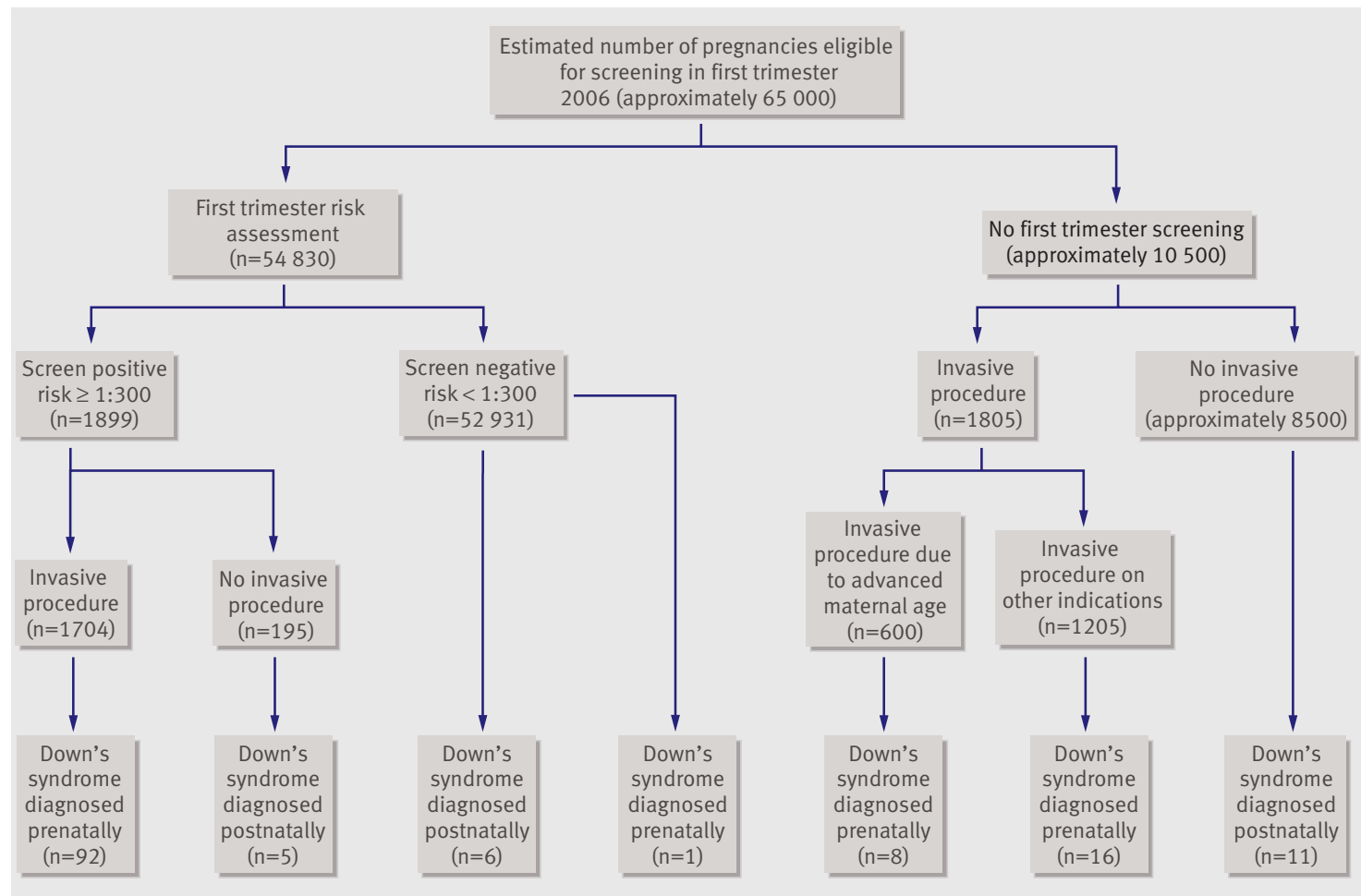

Fig 5 Number of fetuses and newborn infants with Down's syndrome diagnosed prenatally or postnatally according to screening results in Denmark, 2006. Invasive procedures are chorionic villus samplings or amniocenteses

were close to those reported, whereas in 2006 the reported number was lower than expected. This may be due to chance fluctuation, as we believe follow-up is complete. The follow-up time for the numbers reported from 2006 is, however, relatively short and a few cases may therefore still be reported.

In 2005 national screening was not fully implemented. One third of the women were either not offered screening or declined. These women gave birth to a total of 17 infants with Down's syndrome. In 2006 the proportion of non-screened women decreased to $15 \%$, as screening was then available for most women. About 8500 women who were not offered screening or declined screening or a diagnostic test in 2006 gave birth to a total of 11 infants with Down's syndrome. The national guidelines on prenatal screening emphasise that risk assessment for Down's syndrome should be done only if women choose the test on the basis of an informed choice. Therefore despite the programme now being accessible to all pregnant women in Denmark, it is expected that a proportion will still choose not to be screened. The size of this proportion when screening is fully available remains to be established; however, in 2005 only $2 \%$ of the population in two counties declined screening. ${ }^{5}$ Studies on Danish women's attitude, knowledge about screening, and choice of test are ongoing. ${ }^{6}$

We found that the number of prenatal diagnostic tests (chorionic villus samplings and amniocenteses) carried out yearly decreased by more than $50 \%$ during 2000-6. A decrease in the number of prenatal diagnostic procedures could be seen even before the policy was changed, probably because pregnant women became aware of alternative prenatal investigations such as nuchal translucency scanning (fig 3). This was certainly the case in and around Copenhagen, when a prospective study of around 10000 women was done in 1998-2001. ${ }^{7}$ Nuchal translucency scanning was introduced in some departments even before the national guidelines were changed.

In 2005 and 2006 about $3 \%$ of women still had an invasive diagnostic procedure done because of indications other than a screen positive test result, with a tendency towards a reduced number of tests from 2005 to 2006 (2274 women in 2005,1805 in 2006). The decrease was mainly due to fewer women choosing invasive diagnostic tests on the basis of advanced maternal age, as 980 invasive procedures were carried out for that indication in 2005 but decreased to 600 in 2006 . The relatively high number of women choosing chorionic villus sampling or amniocentesis was probably partly due to lack of implementation of the new screening programme. It is also possible that women who had a diagnostic procedure for a previous pregnancy because they were aged 35 or more may have requested a diagnostic test again. When the new screening strategy based on ultrasound and biochemistry has been available for some years we expect the number of invasive diagnostic tests done because of advanced maternal age to decrease even further.

We found that $10-20 \%$ of women with a screen positive test result did not undergo an invasive diagnostic test. This is in accordance with reports from the Copenhagen First Trimester Study. ${ }^{7}$ For 


\section{WHAT IS ALREADY KNOWN ON THIS TOPIC}

Many countries are currently trying to achieve national screening strategies for Down's syndrome

None has described how a combined screening strategy in the first trimester affects numbers of infants born with Down's syndrome or rate of invasive procedures

Detection rates and false positive rates for the combined first trimester risk assessment have been reported only from specialised centres or from regional experience

\section{WHAT THIS STUDY ADDS}

After implementation of a national screening policy in Denmark, the number of infants born with Down's syndrome and the rate of invasive procedures was noticeably reduced

The screening strategy achieved high detection rates and low false positive rates

various reasons (advanced maternal age, conception by assisted reproduction technologies, or risk near the cut-off) some women do not want an invasive diagnostic test, probably because of the associated risk of miscarriage.

The difference in odds of carrying a fetus with Down's syndrome for those who were tested because of a screen positive risk assessment (1:16 in 2005, 1:20 in 2006) compared with that of being tested because of advanced maternal age (1:65 in 2005, 1:75 in 2006) clearly illustrates the rationale in screening using a combined risk assessment in the first trimester. As expected, this strategy reduces the number of unnecessary diagnostic procedures. The procedure related risk of miscarriage after chorionic villus sampling or amniocentesis is reported to be $1 \% .^{8}$ In the group of women having an invasive diagnostic test done because of advanced maternal age in 2005 and 2006 16 chromosomally normal fetuses would then have been miscarried to diagnose 23 cases of Down's syndrome. This should be compared with the 31 fetuses possibly miscarried to diagnose 193 cases of Down's syndrome in the group of women with a screen positive test result. Combined risk assessment in the first trimester is not only a more effective screening method than maternal age alone, it also reduces the risk of miscarrying chromosomally normal fetuses when used as reason to be referred for testing instead of maternal age. Thus the false positive rate of prenatal diagnostic testing has been much reduced by changing the selection criterion from maternal age to risk assessment in the first trimester. The false negative rate has also changed: previously those women who chose to have chorionic villus sampling or amniocentesis because of advanced maternal age had a diagnostic test. Currently women choose to have a screening test; 0.4 women per 1000 in 2005 and 0.1 per 1000 in 2006 subsequently delivered a child with Down's syndrome, despite having a risk assessment below the 1:300 cutoff. These few women may feel more resentment towards the system that failed them than those women who chose not to have an invasive diagnostic test because of advanced maternal age. This emphasises the importance of informing all women about the limitations of screening.
For false positive rates of $3.9 \%$ and $3.3 \%$ in the screened populations we found detection rates for Down's syndrome of $86 \%$ in 2005 and $93 \%$ in 2006 . This is in accordance with the screening performance expected by the Danish National Board of Health when it decided to implement this new screening strategy. This performance may be considered high, especially as the programme in 2005 and 2006 in many centres used a completely new screening method. Furthermore, we report the result of routine clinical practice, where not all risk assessments are based on the optimal variables (combination of maternal age, nuchal translucency scan, and biochemistry) as some are given only on maternal age and nuchal translucency scan or biochemistry. Other authors have also reported screening results achieved in routine clinical practice in up to 13 centres, with detection rates between $83 \%$ and $93 \%$ and false positive rates between $3.9 \%$ and $5.9 \%{ }^{9-13}$ One study collected data from 44 centres in the Netherlands and found a detection rate of $71 \%$ for a false positive rate of $4.7 \% .{ }^{14}$ The authors explain the relatively low detection rate by too small measurements used for nuchal translucency, and expect to improve the detection rate by establishing quality assurance on the measurements. In a large prospective multicentre study the detection rate using a combined screening programme in the first trimester was $92.6 \%$ for a false positive rate of $5.2 \% .{ }^{15}$ Our data show that it is possible to introduce this screening strategy in as many as 19 different centres and still obtain national detection and false positive rates similar to those from specialised centres.

It is well known that implementation of new screening strategies requires effort, and many countries are currently facing various problems in trying to achieve a national strategy. ${ }^{16-18}$ In Denmark, with its public, free of charge hospital system, we have succeeded in establishing a strong national organisation for fetal medicine. Recruitment and training of sonographers as well as quality control are in accordance with the guidelines from the Fetal Medicine Foundation in London (www.fetalmedicine.com). We have implemented national guidelines on screening in the first trimester, and from 1 January 2007 a common cut-off of 1:300 for referral to invasive diagnostic testing at the time of screening. Furthermore, the use of the same database software in all departments allows national data to be merged. A national quality database has been established that merges data from all fetal medicine units, the Danish national cytogenetic registry, and the national patient registry. This will allow follow-up of all screened women at a national level, as well as monitoring of detection rates, false positive rates, and invasive testing rates, a quality control that is considered essential after the implementation of a new screening programme.

Members of the Danish Fetal Medicine Research Group are: Hans Jakob Andersen, Jeanette Christensen, Vibeke Ersbak, Richard Farlie, Carsten Henriques, Annette Wind Olesen, Anni Holmskov, Lisa Neerup Jensen, Anette Kristiansen, Torben Larsen, Hedvig Poulsen, Jan Ramb, Lillian Skibsted, Peter Skovbo, Steffen Sommer, Lene Sperling, Susanne 
Vemmelund Juul, Helle Zingenberg. We thank Jan Hansen for retrieval of data from the Danish Central Cytogenetic Registry, Kirsten Rasmussen for retrieval of data from the Genetic Database of Funen, Steen Rasmussen (Danish National Board of Health) for providing gestational age at delivery in cases where no screening was done,and the departments of clinical biochemistry for analysing the biochemical samples used in the screening programme.

Contributors: AT and CKE planned and did the study in cooperation with FSJ, OBP, and KS. CKE and AT wrote the manuscript and are guarantors. Hans Jakob Andersen, Jeanette Christensen, Vibeke Ersbak, Richard Farlie, Carsten Henriques, Annette Wind Olesen, Anni Holmskov, Lisa Neerup Jensen, FSJ, Anette Kristiansen, Torben Larsen, OBP, Hedvig Poulsen, Jan Ramb, Lillian Skibsted, Peter Skovbo, Steffen Sommer, Lene Sperling, KS, Susanne Vemmelund Juul and Helle Zingenberg retrieved data from the local Astraia servers and reviewed and accepted the final manuscript. Funding: None.

Competing interests: None declared

Ethical approval: This study was approved by the Danish Data Protection Agency and Danish National Board of Health.

Provenance and peer review: Not commissioned; externally peer reviewed.

1 Danish National Board of Health. Report from a working commission "Prenatal diagnosis and risk assessment." www.sst.dk/upload/ fosterdiagnostik1_001.pdf. 2003.

2 Jorgensen FS. [Organization of obstetric ultrasound in Denmark 2000 Description of the development since 1990]. Ugeskr Laeger 2003;165:4404-9.

3 Morris JK, Mutton DE, Alberman E. Revised estimates of the maternal age specific live birth prevalence of Down's syndrome. J Med Screen 2002;9:2-6.

4 Snijders RJ, Sundberg K, Holzgreve W, Henry G, Nicolaides KH. Maternal age- and gestation-specific risk for trisomy 21. Ultrasound Obstet Gynecol 1999;13:167-70.

5 Torring N, Jolving LR, Petersen OB, Holmskov A, Hertz JM, Uldbjerg N. [Prenatal diagnostics in Arhus and Viborg counties after implementation of first trimester risk assessment]. Ugeskr Laeger 2008;170:50-4.

6 Bangsgaard L, Tabor A. OC30: Are pregnant women and their partners making an informed choice about first-trimester risk assessment for Down syndrome? Ultrasound Obstet Gynecol 2007;30:376.

7 Wojdemann KR, Shalmi AC, Christiansen M, Larsen SO, Sundberg K, Brocks V, et al. Improved first-trimester Down syndrome screening performance by lowering the false-positive rate: a prospective study of 9941 low-risk women. Ultrasound Obstet Gynecol 2005;25:227-33.

8 Alfirevic Z, Sundberg K, Brigham S. Amniocentesis and chorionic villus sampling for prenatal diagnosis. Cochrane Database Syst Rev 2003;(3):CD003252.

9 Hadlow NC, Hewitt BG, Dickinson JE, Jacoby P, Bower C. Communitybased screening for Down's syndrome in the first trimester using ultrasound and maternal serum biochemistry. Br J Obstet Gynaecol 2005;112:1561-4.

10 O'Leary P, Breheny N, Dickinson JE, Bower C, Goldblatt J, Hewitt B, et al. First-trimester combined screening for Down syndrome and other fetal anomalies. Obstet Gynecol 2006;107:869-76

11 Soergel P, Pruggmayer M, Schwerdtfeger R, Muhlhaus K, Scharf A. Screening for trisomy 21 with maternal age, fetal nuchal translucency and maternal serum biochemistry at 11-14 weeks: a regiona experience from Germany. Fetal Diagn Ther 2006;21:264-8.

12 Spencer K, Spencer CE, Power M, Dawson C, Nicolaides KH. Screening for chromosomal abnormalities in the first trimester using ultrasound and maternal serum biochemistry in a one-stop clinic: a review of three years prospective experience. Br J Obstet Gynaecol 2003;110:281-6.

13 Stenhouse EJ, Crossley JA, Aitken DA, Brogan K, Cameron AD, Connor JM. First-trimester combined ultrasound and biochemical screening for Down syndrome in routine clinical practice. Prenat Diagn 2004;24:774-80.

14 Schielen PC, van Leeuwen-Spruijt M, Belmouden I, Elvers LH, lonker M, Loeber JG. Multi-centre first-trimester screening for Down syndrome in the Netherlands in routine clinical practice. Prenat Diagn 2006;26:711-8

15 Nicolaides KH, Spencer K, Avgidou K, Faiola S, Falcon O. Multicenter study of first-trimester screening for trisomy 21 in 75821 pregnancies: results and estimation of the potential impact of individual risk-orientated two-stage first-trimester screening. Ultrasound Obstet Gynecol 2005;25:221-6.

16 Chang TC. Antenatal screening for Down syndrome in New Zealand: time for a national screening policy? Aust N Z J Obstet Gynaecol 2006;46:92-6

17 O'Leary P, Breheny N, Reid G, Charles T, Emery]. Regional variations in prenatal screening across Australia: stepping towards a national policy framework. Aust N Z J Obstet Gynaecol 2006;46:427-32.

18 Reddy UM, Mennuti MT. Incorporating first-trimester Down syndrome studies into prenatal screening: executive summary of the National Institute of Child Health and Human Development workshop. Obstet Gynecol 2006;107:167-73.

Accepted: 30 September 2008 